\title{
IS6110-based Differentiation of Mycobacterium tuberculosis Strains
}

\author{
ANNA ŻACZEK¹, MAŁGORZATA ZIÓŁKIEWICZ², ARKADIUSZ WOJTASIK², \\ JAROSŁAW DZIADEK ${ }^{1,2}$ and ANNA SAJDUDA ${ }^{3 *}$ \\ ${ }^{1}$ Department of Biochemistry and Cell Biology, Faculty of Biology and Agriculture, \\ University of Rzeszów, Ćwiklińskiej 2, 35-601 Rzeszów, Poland \\ ${ }^{2}$ Institute of Medical Biology, Polish Academy of Sciences, Lodowa 106, 93-232 Łódź, Poland \\ ${ }^{3}$ Department of Genetics of Microorganisms, Faculty of Biology and Environmental Protection, \\ University of Łódź, Banacha 12/16, 90-237 Łódź, Poland
}

Received 15 January 2013, accepted 3 April 2013

Abstract

In this study, 62 Mycobacterium tuberculosis strains were characterized by fast ligation-mediated PCR (FLiP) and, previously performed, IS6110 restriction fragment length polymorphism (RFLP). FLiP proved a reproducible and specific method for differentiation between M. tuberculosis strains. The discriminatory power of FLiP was close to that of the reference IS6110 RFLP suggesting its usefulness in studying the genetic diversity of M. tuberculosis strains.

Key words: M. tuberculosis, fast ligation-mediated PCR, IS6110, RFLP

Mycobacterium tuberculosis is the causative agent of tuberculosis (TB) that remains a major infectious disease and causes high morbidity and mortality worldwide (World Health Organization, 2010). To increase our understanding of the epidemiology of TB, molecular typing methods based on various genetic markers have been developed in recent years (Cave et al., 2005). The current international standard for epidemiological typing of $M$. tuberculosis is restriction fragment length polymorphism (RFLP) method based on the variability at a genomic location and the number of copies of the insertion element IS6110 between strains (van Embden et al., 1993). The IS6110 RFLP provides best resolution at the population level (Mathema et al., 2006). However, it suffers from some limitations including its labor intensiveness and the requirement of a large quantity (at least $2 \mu \mathrm{g}$ ) of purified genomic DNA (Behr and Mostowy, 2007). In this context, alternative PCRbased methods, including fast ligation-mediated PCR (FLiP) (Reisig et al., 2005), seem particularly promising. FLiP is a modified mixed-linker PCR, a rapid typing method based on the PCR amplification of RFLP fragments containing the 3' end of IS6110 (Haas et al., 1993). It favorably compared with eight other novel typing methods for M. tuberculosis complex according to discriminatory power and reproducibility (Kremer et al., 2005), and was suggested as a first-line test prior to high-throughput analysis or standard IS6110 RFLP as well as a secondary typing technique for other, nonIS6110-based methods (Reisig et al., 2005).

In the present study, we applied FLiP analysis for differentiation of $62 \mathrm{M}$. tuberculosis strains, previously characterized by the reference IS6110 RFLP (Krawczyk et al., 2011), and compared the discriminatory ability of both methods used. Also, the specificity of FLiP amplicons was determined.

The $62 \mathrm{M}$. tuberculosis strains analyzed here were obtained in 2006-2008 from TB patients hospitalized in Łódź, Poland. All strains were previously characterized by IS6110 RFLP analysis using the internationally standardized protocol (van Embden et al., 1993; Krawczyk et al., 2011). Purified chromosomal DNA from RFLP typing was available for further investigation by FLiP. The analysis was performed as originally described by Reisig et al. (2005).

The fingerprint patterns obtained by both IS6110 based methods were analyzed by using BioNumerics software, version 5.0 (Applied Maths, Sint-MartensLatem, Belgium). Dendrograms were generated based on the unweighted pair group method with arithmetic averages (UPGMA) and Dice similarity coefficient.

The Hunter-Gaston Discriminatory Index (HGDI) (Hunter and Gaston, 1988) was used as a numerical index for the discriminatory power of each typing method.

\footnotetext{
* Corresponding author: A. Sajduda, Department of Genetics of Microorganisms, Faculty of Biology and Environmental Protection, University of Łódź, Banacha 12/16, 90-237 Łódź, Poland; phone: +48 4263547 72; fax: +48 4266558 18; e-mail: asajduda@biol.uni.lodz.pl
} 


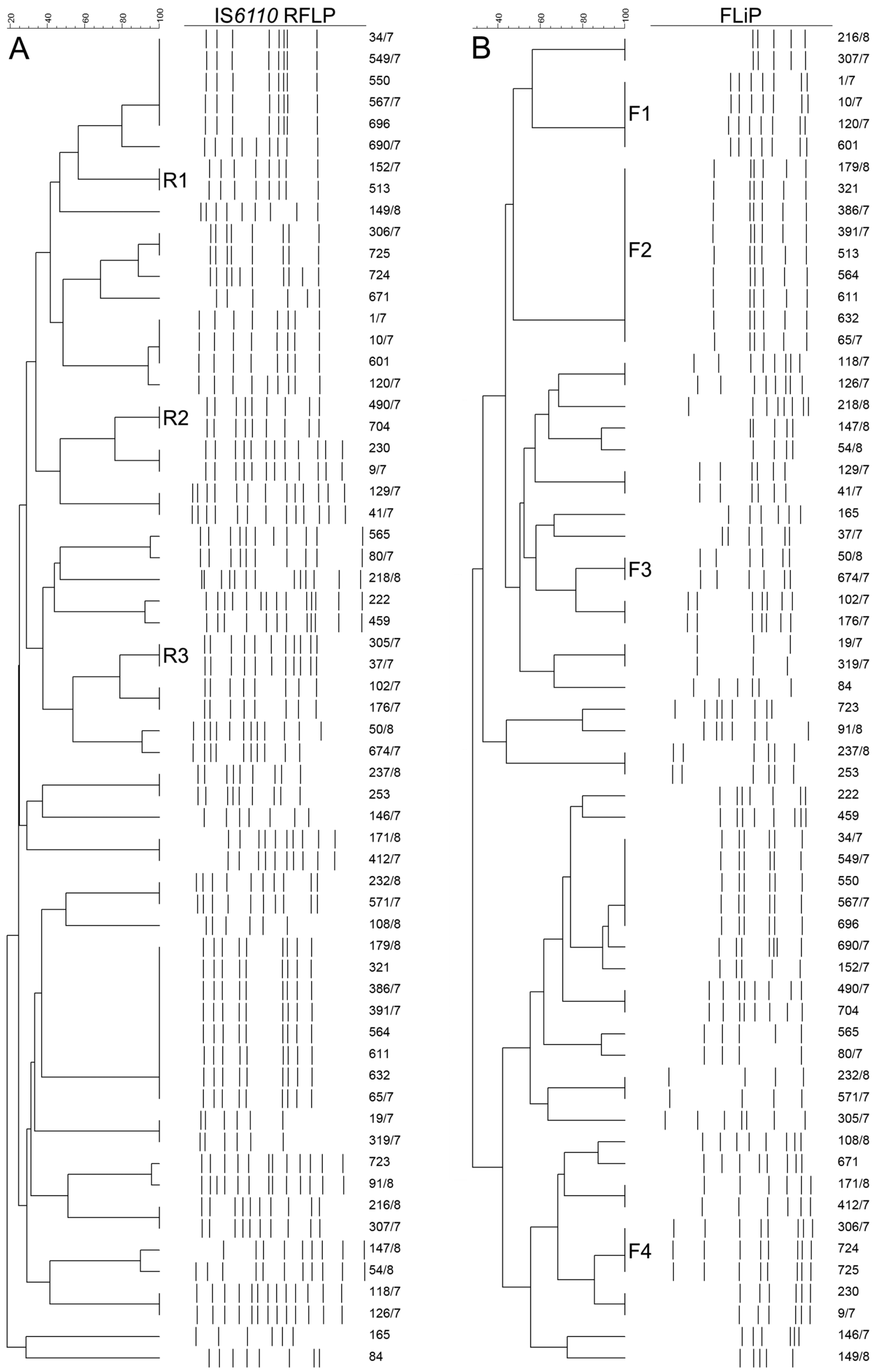


FLiP amplification products obtained with DNA of the strain M. tuberculosis 391/7 were blunted and cloned into pJET1.2 (Fermentas Life Sciences). The recombined vectors carrying inserts of different sizes were selected for sequencing. Both strands of the cloned FLiP amplification products were sequenced by Genomed S.A., Warsaw, Poland. The obtained sequences were compared with those deposited in the GenBank database by using BLAST (http://www.ncbi.nlm.nih.gov/BLAST/).

FLiP is a relatively novel DNA typing method for M. tuberculosis complex strains (Reisig et al., 2005). Based on the original mixed-linker approach (Haas et al., 1993), it is a simplified and faster method which allows IS6110 strain typing within $6.5 \mathrm{~h}$, starting from purified mycobacterial DNA or crude cell lysates. Kremer et al. (2005), in an extensive interlaboratory study comparing nine recently described PCR-based assays, concluded that VNTR (variable numbers of tandem repeat) and FLiP are both rapid, highly reliable, and discriminative epidemiological typing methods for M. tuberculosis. However, despite its potential to reliably differentiate between strains, FLiP has not been widely used since its publication.

In this context, the present study reports a molecular characterization of M. tuberculosis isolated in Łódź, Poland through genotyping by FLiP in comparison with previously performed IS6110 RFLP typing (Krawczyk et al., 2011). The copy number of IS6110, determined by the reference method in each of the 62 strains, ranged from 6 to 14 (Fig. 1A). The majority, 40 (64.5\%) of the strains contained 8-10 copies, with a mean of 9 bands that is consistent with our previous study of $M$. tuberculosis in Łódź (Dela et al., 2006). In contrast, the number of bands in the FLiP patterns varied from 3 to 8 , with an average of 6 bands (Fig. 1B). The vast majority, 56 (90\%) of the patterns contained 1-8 bands less compared to the IS6110 RFLP profiles of the respective strains. These results indicate that the number of bands in the FLiP DNA fingerprints may not necessarily reflect the number of IS6110 copies in the strains tested. The observed differences could either be caused by the visual interpretation of the patterns, by failure of the polyacrylamide gels to sufficiently separate the PCR fragments, or by difficulties of the PCR itself. Nevertheless, FLiP typing proved highly reproducible confirming previous observations (Kremer et al., 2005). The reproducibility of FLiP was assessed by including triplicate DNA samples of M. tuberculosis H37Rv and 16 test strains (data not shown).

FLiP typing differentiated 35 patterns among the 62 M. tuberculosis strains analyzed (Fig. 1B). Of the
35 patterns, 21 were unique, whereas the remaining 14 cluster patterns included 41 (66\%) strains. One cluster occurred in nine strains with identical FLiP DNA fingerprints, three clusters comprised five, four and three strains, respectively, and 10 clusters were found in two strains each. The IS6110 RFLP typing differentiated 36 patterns among this strain set. The level of differentiation of FLiP was slightly lower compared to that of IS6110 RFLP and there were some discordant results between the two methods. IS6110 RFLP differentiated strains (50/8 and 674/7) in the FLiP cluster F3 (Fig. 1). Also, strains 120/7 and 724 had slightly different IS6110 RFLP patterns compared to three or two other strains in the respective clusters F1 and F4. On the other hand, four strains clustered by IS6110 RFLP in two patterns R2 and R3 were found slightly (strains 490/7 and 704, cluster R2) or totally (strains 305/7 and 37/7, cluster R3) different in the FLiP analysis (Fig. 1). Strains in cluster R3 were previously differentiated also by spoligotyping (Krawczyk et al., 2011). Moreover, FLiP discriminated the strains in cluster R1. However, only strain 152/7 was unique, while the strain 513 clustered together with eight others (Fig. 1B, pattern F2). Unfortunately, it was not possible to verify the aforementioned discordant patterns due to the lack of sufficient epidemiological data associated with them.

Clustering results from both IS6110-based typing methods applied in the present study were compared to determine their discriminatory power. FLiP typing gave resolving power close to that of the reference method (HGDI $=0.966$ and 0.971 , respectively). The intrinsically lower band numbers of FLiP patterns might contribute to the slightly lower resolution of this method as compared with the reference IS6110 RFLP. A broad review of literature, with regard to degree of differentiation of various molecular methods used in the epidemiology of $M$. tuberculosis, demonstrated a higher discriminatory power for FLiP (HGDI = 0.9945) in comparison with the mean value for IS6110 RFLP obtained in various studies (HGDI $=0.9515$ or 0.9808 , when the lowest value obtained was rejected) (Rozo-Anaya and Ribón, 2010). However, this could be due to the application of FLiP only in one study (Reisig et al., 2005), while there is a bulk of published IS6110 RFLP data, including some studies where a low number of strains was analyzed, that resulted in lower HGDI and reduced the overall mean value for the reference method.

It has been shown, that in PCR-based DNA fingerprinting patterns some of the products could result from nonspecific amplification (Kremer et al., 2005). Reisig etal. (2005) demonstrated the specificity of

Fig. 1. IS6110 RFLP (A) and FLiP (B) patterns of the $62 \mathrm{M}$. tuberculosis strains and the corresponding dendrograms. The numbers of strains and selected patterns are indicated at the sides of the lane maps displaying the DNA fingerprints of the analyzed strains. The similarity among the patterns is indicated as a percentage above the dendrograms. 


\begin{tabular}{|c|c|c|}
\hline & A & $\mathrm{B}$ \\
\hline & 3606 & 3666 \\
\hline Mtb391/7 & GGTCTCAGATCAGAGAGTCTCCGGACTCACCGGGGCGGTCA & ATAGCTATGCGCCGTITGAGAACGIACAITITGIGATTGCGGITCGCATITAGGAGCCCGG \\
\hline $\begin{array}{l}\text { MtbT46 } \\
\text { IS6110-3' }\end{array}$ & , GGTCTCAGATCAGAGAGTCTCCGGACTCACCGGGGCGGTTCA & $\begin{array}{l}\text { TAGCTATGCGCCGTTTGAGAACGIACATITGTGATTGCGGITCGCATITAGGAGCCCG } \\
\text { G }\end{array}$ \\
\hline
\end{tabular}

Fig. 2. Alignment of partial DNA sequence of an exemplary FLiP-amplified product in M. tuberculosis 391/7, a GenBank-derived sequence of M. tuberculosis T46 cont. 1.52, and the 3' end of IS6110. Nucleotides common to both M. tuberculosis strains are written in bold (B). A, 3' end of IS6110.

FLiP-amplified patterns by hybridization of DNA fragments with the diagnostic oligonucleotide, containing part of the IS6110 sequence. In the present study, FLiP fragments from the pattern of M. tuberculosis 391/7, representing the largest cluster of strains, were cloned into a plasmid vector PJET1.2 and their DNA sequences were determined. After comparison of the sequences obtained with those available in the GenBank database, all fragments were shown to contain the 3 ' end of IS6110 - that harbors the transposase gene - and M. tuberculosis-specific nucleotide sequence (Fig.2), demonstrating the specificity of FLiP and its potential application in mapping genomic locations of IS6110 in M. tuberculosis strains.

In summary, FLiP proved a reproducible and specific method for differentiation of M.tuberculosis strains, showing a discriminatory power comparable to that of the IS6110 RFLP. Based on the previous and present results, FLiP analysis seems a valuable adjunct to the reference method. However, to further confirm its usefulness in typing $M$. tuberculosis, its evaluation on a larger number of strains from various geographical regions would be necessary.

\section{Acknowledgements}

This work was supported by grant from the National Science Centre (NCN, project no. N N302 111338), and also partially performed within the project "Centre of Applied Biotechnology and Basic Sciences" (no. POPW.01.03.00-18-018/09) supported by the Operational Program "Development of Eastern Poland 2007-2013".

\section{Literature}

Behr M.A. and S. Mostowy. 2007. Molecular tools for typing and branding the tubercle bacillus. Curr. Mol. Med. 7: 309-317.
Cave M.D., M. Murray and E. Nardell. 2005. Molecular epidemiology of Mycobacterium tuberculosis, pp. 33-46. In: Cole S.T., K.D. Eisenach, D.N. McMurray and W.R. Jacobs, Jr. (eds.). Tuberculosis and the Tubercle Bacillus. ASM Press, Washington, D.C.

Dela A., A. Sajduda, I. Pawłowska and J. Dziadek. 2006. Molecular characterization of Mycobacterium tuberculosis isolates from Łódź, Poland: analysis by IS6110 restriction fragment length polymorphism and double-repetitive-element PCR. J. Infect. 52: 346-353.

Haas W.H., W.R. Butler, C.L. Woodley and J.T. Crawford. 1993. Mixed-linker polymerase chain reaction: a new method for rapid fingerprinting of isolates of the Mycobacterium tuberculosis complex. J. Clin. Microbiol. 31: 1293-1298.

Hunter P.R. and M.A. Gaston. 1988. Numerical index of the discriminatory ability of typing systems: an application of Simpson's index of diversity. J. Clin. Microbiol. 26: 2465-2466.

Krawczyk M., A. Brzostek, A. Gorna, K. Knapska, M. Ziolkiewicz, A. Wojtasik and J. Dziadek. 2011. Epidemiological analysis of Mycobacterium tuberculosis strains isolated in Lodz, Poland. Int. J. Tuberc. Lung Dis. 15: 1252-1258.

Kremer K., C. Arnold, A. Cataldi, M.C. Gutiérrez, W.H. Haas, S. Panaiotov, R.A. Skuce, P. Supply, A.G.M. van der Zanden and D. van Soolingen. 2005. Discriminatory power and reproducibility of novel DNA typing methods for Mycobacterium tuberculosis complex strains. J. Clin. Microbiol. 43: 5628-5638.

Mathema B., N.E. Kurepina, P.J. Bifani and B.N. Kreiswirth. 2006. Molecular epidemiology of tuberculosis: current insights. Clin. Microbiol. Rev. 19: 658-685.

Reisig F., K. Kremer, B. Amthor, D. van Soolingen and W.H. Haas. 2005. Fast ligation-mediated PCR, a fast and reliable method for IS6110-based typing of Mycobacterium tuberculosis complex. J. Clin. Microbiol. 43: 5622-5627.

Rozo-Anaya J.C. and W. Ribón. 2010. Molecular tools for Mycobacterium tuberculosis genotyping. Rev. salud pública 12: 510-521. van Embden J.D., M.D. Cave, J.T. Crawford, B. Gicquel, P. Hermans, R. McAdam, T. Shinnick and P.M. Small. 1993. Strain identification of Mycobacterium tuberculosis by DNA fingerprinting: recommendations for a standardized methodology. J. Clin. Microbiol. 31: 406-409.

World Health Organization. 2010. Global tuberculosis control: WHO Report 2010. WHO Press, Geneva. 